\title{
One step closer to finding the Fountain of Youth in our muscles: can we grow old while staying young at heart?
}

\section{Sveva Bollini*}

Department of Experimental Medicine (DIMES), University of Genova, Via A. Pastore 3, 16132 Genova, Italy

\section{Commentary on 'Aging disrupts muscle stem cell function by impairing matricellular WISP1 secretion from fibro-adipogenic progenitors' by L. Lukjanenko et al., Cell Stem cell, $2019{ }^{1}$}

Senectus ipsa morbus est [Publius Terentius Afer: Phormio: a. IV, (160 B.C.)]: ageing is itself a disease. The quest of eternal youth has always been a major dream for humanity, from mythology up to current science. People worldwide are living longer with the population over 60 years old raising up to 2 billion by $2050 .^{2}$ Thus, the pursuit for a molecular 'holy grail' for ageing, if not healthy, at least smarter, is a medical demand.

The decline of working potential and regenerative properties is main hallmarks of tissue aging. The skeletal muscle healing restorative capacity relies on activation of resident progenitors, the muscle stem cells (MuSCs), which become less responsive and dysregulated as getting elderly. ${ }^{3}$ Recently, Lukjanenko et al. ${ }^{1}$ elegantly addressed ageing detrimental influence on MuSCs in mice. Authors focused on the functional paracrine cross-talk between MuSCs and their regulatory cells in the skeletal muscle niche, namely fibro-adipogenic precursors (FAPs). Ageing significantly affects FAPs, as they fail to support MuSC renewal and differentiation and increase their pro-fibrotic commitment after muscle injury. On the contrary, young FAPs modulate the muscle healing system by stronger paracrine effects on MuSCs. Authors pin-pointed a molecular candidate in WISP1 (Wnt1 Inducible Signalling pathway Protein 1) within the secretome (i.e. the whole of cell-secreted paracrine molecules) of the injuryactivated and young FAPs, as further validated in WISP ${ }^{-/-}$mice with agerelated defects in muscle regeneration; paracrine therapy via systemic administration of recombinant WISP1 in aged mice showed to restore regenerative MuSC myogenesis. Therefore, targeting the signalling cross-talk within the MuSC niche via the FAP secretome, could help rejuvenating muscle healing, while counteracting age-related decay of regenerative potential.

This study provides relevant insights for cardiac repair and regeneration as well. Ageing is a leading factor for cardiovascular disease, with the heart long being considered devoid of any regenerative capacity. Disease- or injury-related compensatory mechanisms include hypertrophy of resident cardiomyocytes and organ-wide remodelling, leading to heart failure. In the last years, growing interest has been dedicated to the identification of endogenous myocardial precursors with responsive restorative potential. Several populations of cardiac progenitor cells (CPCs) have been described with putative cardiovascular differentiation capacity; despite initial enthusiasm, an increasing body of works has been questioning the efficacy of CPC myogenic potential following injury. Indeed, in the adult injured heart, CPCs have shown to preferentially commit to a fibroblastic or vascular phenotype, with the few newly generated cardiomyocytes deriving from surviving pre-existing ones. ${ }^{4}$ Deutsch et al. ${ }^{5}$ clearly showed that myocardial infarction triggers resident murine cardiac stromal cells expressing an activated enhancer for the multipotent progenitor embryonic gene $N k x 2.5$. Although these cells retrieve expression of stem cell- and early cardiac transcription factors, they undertake a pro-fibrotic fate, rather than differentiating into new cardiomyocytes, suggesting that genetic restoration of the embryonic programme during adulthood might not be sufficient to reinstate developmental plasticity. Yet, CPCs have demonstrated to instruct the cardiac microenvironment by modulatory signalling and support local angiogenesis after injury ${ }^{6}$; ex vivo administration of the CPC secretome (including extracellular vesicles) in rodent models of myocardial infarction results in cardiac function improvement with preservation of viable cardiomyocytes. ${ }^{7}$ Hence, CPCs are likely to be considered endogenous regulatory supporting cells, rather than myogenic precursors; they are primed by a fibrogenic signature and endowed with relevant secretory potential, thus resembling the cardiac counterpart to skeletal muscle FAPs.

Mammal cardiomyocytes become post-mitotic during the complete maturation from peri-natal age into adulthood, with meagre turnover potential during life. In contrast, the early neonatal mouse myocardium maintains efficient regeneration potential after extensive damage via dedifferentiation and proliferation of pre-existing cardiomyocytes; however, this restorative response is strictly limited to the first 7 days after birth, with clear transition to fibrosis/scarring soon afterwards. ${ }^{8}$ The prompt peri-natal cardiomyocyte withdrawal from cell cycle into the differentiated resting state implies a drastic 'memory loss' of endogenous regenerative mechanisms, as cells 'age' into the mature functional phenotype. Emerging data have indicated some putative candidate targets to retrieve and 'rejuvenate' resident cardiomyocyte proliferative potential, such as the activation of cell cycle regulators or the inhibition of GSK-3 kinase signalling, a sensitive regulator of mammalian myocardial renewal. ${ }^{9}$

\footnotetext{
* Corresponding author. Tel: +39 010353 38080/+39 010555 8257; fax: +39 010555 8241, E-mail: sveva.bollini@unige.it

Published on behalf of the European Society of Cardiology. All rights reserved. (c) The Author(s) 2019. For permissions, please email: journals.permissions@oup.com.
} 
Notably, during cardiogenesis epicardial CPC provide paracrine signalling to the developing myocardium by means of mitogens influencing cardiomyocyte renewal; embryonic epicardial CPC contribute to cardiac fibroblasts, which support cardiomyocyte expansion; conversely, adult cardiac fibroblasts drive cardiomyocyte hypertrophy. ${ }^{10}$ Such close crosstalk between cardiomyocytes and pro-fibrogenic CPC strongly resembles the paracrine relationship of skeletal muscle FAPs and MuSCs. Since WISP1 has been shown to play a crucial role in muscle regeneration, evaluation on its paracrine potential on the CPC-cardiomyocyte axis might offer valuable understandings for myocardial regeneration. Human recombinant WISP1 has been described to exert cardioprotective effects on isolated adult mouse cardiomyocytes experiencing anthracycline-induced cardiotoxicity, via inhibition of GSK-3 $\beta$ phosphorylation with $\beta$-catenin nuclear translocation. ${ }^{11}$ Pharmacological blocking of GSK-3 promotes proliferation in human neonatal cardiomyocytes by up-regulating cell cycle and cytokinesis mechanisms via canonical Wnt pathway ${ }^{12}$; thus, it is reasonable to think that WISP1 might also impact myocardial renewal. In stark contrast, adult rodents infused with Angiotensin-II show increased WISP1 expression in proliferative cardiac fibroblasts resulting in myocardial hypertrophy and activation of fibrosis. ${ }^{13}$ By acting as pro-mitogenic and pro-survival paracrine effector by cardiac cell type-specific manner, WISP1 exerts complex biological effects, acting as friend or foe. Therefore, it might be interesting to assess its possible role in rejuvenating adult cardiomyocyte cell cycle progression when secreted by embryonic/peri-natal CPC compared to adult ones.

Lukjanenko et al. also demonstrated that skeletal muscle FAPs modulate the expression of the PDGFR $\alpha$ gene intronic variant $(P D G F R \alpha-I n)$ as they age; $P D G F R \alpha-$ In prevents FAP skewing to fibrotic cells and it gets reduced in elderly and failing progenitors. Endogenous $\mathrm{CPC}$ have been described as PDGFR $\alpha$-positive stromal cells, ${ }^{14}$ contributing to ventricle fibrofatty scarring when impaired during arrhythmogenic cardiomyopathy. ${ }^{15}$ Then, profiling PDGFR $\alpha$-In modulation during CPC ageing could offer meaningful information as therapeutic target to limit unnecessary fibrosis.

This body of work endorses the relevance of the paracrine modulation of the tissue microenvironment to inhibit functional ageing. Tissue rejuvenation might be pursued by stimulating endogenous cells via suitable paracrine instruction, rather than by coercing their genome by means of reprogramming or by replacing them through cell transplantation. Since increasing evidences indicates that restoration of resident cardiomyocyte proliferation represents the primary therapeutic mechanism for heart regeneration, results from Lukjanenko et al. might offer useful insights in the cardiac scenario. Rejuvenation of the CPC secretome might offer a putative working strategy to unlock resident cardiomyocyte renewal and provide fertile soil for a good seed. Hence, reinstating the developmental CPC-cardiomyocyte molecular cross-talk during adulthood might help growing old, while staying young at heart.

\section{Conflict of interest: none declared.}

\section{References}

1. Lukjanenko L, Karaz S, Stuelsatz P, Gurriaran-Rodriguez U, Michaud J, Dammone G, Sizzano F, Mashinchian O, Ancel S, Migliavacca E, Liot S, Jacot G, Metairon S, Raymond F, Descombes P, Palini A, Chazaud B, Rudnicki MA, Bentzinger CF, Feige JN. Aging disrupts muscle stem cell function by impairing matricellular WISP1 secretion from fibro-adipogenic progenitors. Cell Stem Cell 2019;24:433-446.e7.

2. World Health Organization Ageing and Health. https://www.who.int/news-room/ fact-sheets/detail/ageing-and-health.

3. Feige $P$, Brun CE, Ritso M, Rudnicki MA. Orienting muscle stem cells for regeneration in homeostasis, aging, and disease. Cell Stem Cell 2018;23:653-664.

4. Li Y, He L, Huang X, Bhaloo SI, Zhao H, Zhang S, Pu W, Tian X, Li Y, Liu Q, Yu W, Zhang L, Liu X, Liu K, Tang J, Zhang H, Cai D, Ralf AH, Xu Q, Lui KO, Zhou B. Genetic lineage tracing of nonmyocyte population by dual recombinases. Circulation 2018;138:793-805.

5. Deutsch M-A, Doppler SA, Li X, Lahm H, Santamaria G, Cuda G, Eichhorn S, Ratschiller T, Dzilic E, Dreßen M, Eckart A, Stark K, Massberg S, Bartels A, Rischpler C, Gilsbach R, Hein L, Fleischmann BK, Wu SM, Lange R, Krane M. Reactivation of the Nkx2.5 cardiac enhancer after myocardial infarction does not presage myogenesis. Cardiovasc Res 2018;114:1098-1114.

6. Zhou B, Honor LB, He H, Ma Q, Oh J-H, Butterfield C, Lin R-Z, Melero-Martin JM, Dolmatova E, Duffy HS, von GA, Zhou P, Hu YW, Wang G, Zhang B, Wang L, Hall JL, Moses MA, McGowan FX, Pu WT. Adult mouse epicardium modulates myocardial injury by secreting paracrine factors. J Clin Invest 2011;121:1894-1904.

7. Barile L, Cervio E, Lionetti V, Milano G, Ciullo A, Biemmi V, Bolis S, Altomare C, Matteucci M, Di Silvestre D, Brambilla F, Fertig TE, Torre T, Demertzis S, Mauri P, Moccetti T, Vassalli G. Cardioprotection by cardiac progenitor cell-secreted exosomes: role of pregnancy-associated plasma protein-A. Cardiovasc Res 2018;114: 992-1005.

8. Porrello ER, Mahmoud Al, Simpson E, Hill JA, Richardson JA, Olson EN, Sadek HA. Transient regenerative potential of the neonatal mouse heart. Science 2011;331: 1078-1080.

9. Singh AP, Umbarkar P, Guo Y, Force T, Gupte M, Lal H. Inhibition of GSK-3 to induce cardiomyocyte proliferation: a recipe for in situ cardiac regeneration. Cardiovasc Res 2019;115:20-30.

10. Masters M, Riley PR. The epicardium signals the way towards heart regeneration. Stem Cell Res 2014;13:683-692.

11. Venkatesan B, Prabhu SD, Venkatachalam K, Mummidi S, Valente AJ, Clark RA, Delafontaine P, Chandrasekar B. WNT1-inducible signaling pathway protein-1 activates diverse cell survival pathways and blocks doxorubicin-induced cardiomyocyte death. Cell Signal 2010;22:809-820.

12. Wang S, Ye L, Li M, Liu J, Jiang C, Hong H, Zhu H, Sun Y. GSK-3 $\beta$ Inhibitor CHIR99021 promotes proliferation through upregulating $\beta$-catenin in neonatal atrial human cardiomyocytes. J Cardiovasc Pharmacol 2016;68:425-432.

13. Shanmugam P, Valente AJ, Prabhu SD, Venkatesan B, Yoshida T, Delafontaine P, Chandrasekar B. Angiotensin-II type 1 receptor and NOX2 mediate TCF/LEF and CREB dependent WISP1 induction and cardiomyocyte hypertrophy. J Mol Cell Cardiol 2011;50:928-938.

14. Noseda M, Harada M, McSweeney S, Leja T, Belian E, Stuckey DJ, Abreu Paiva MS, Habib J, Macaulay I, Aj de S, Al-Beidh F, Sampson R, Lumbers RT, Rao P, Harding SE, Blakemore AIF, Eirik Jacobsen S, Barahona M, Schneider MD. PDGFR $\alpha$ demarcates the cardiogenic clonogenic Sca1+ stem/progenitor cell in adult murine myocardium. Nat Commun 2015;6:6930.

15. Paylor B, Fernandes J, McManus B, Rossi F. Tissue-resident Sca1+ PDGFR $\alpha+$ mesenchymal progenitors are the cellular source of fibrofatty infiltration in arrhythmogenic cardiomyopathy. F1000Res 2013;2:141. 


\section{Author}

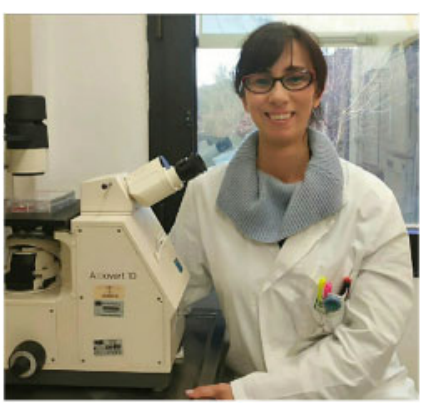

Biography: Dr Sveva Bollini graduated in Medical Biotechnology and got her PhD from the University of Padova in Italy, where she studied the cardiomyogenic differentiation potential of human amniotic fluid stem cells. During her post-doctoral work, she worked on the lineage characterization of reactivated epicardiumderived progenitor cells for cardiac repair under the supervision of Prof. Paul Riley, at UCL-University College London, London and at the University of Oxford, Oxford in UK. In 2014, she was presented with the 'Rita Levi Montalcini' Young Investigator Award from the Italian Ministry of Research and Education (MIUR) and invited back to Italy to study the paracrine potential of the human amniotic fluid stem cell secretome (i.e. the whole of cell-secreted soluble factors and extracellular vesicles) to enhance cardiac repair. Currently, she is a Group Leader and an Associate Professor in Experimental Biology in the Department of Experimental Medicine, University of Genova in Genova, Italy. Her research mainly focuses on the functional characterization of human foetal and peri-natal stem cell secretome to rejuvenate myocardial renewal following injury and to unlock endogenous mechanisms of cardiac regeneration. Dr Bollini is a member of the ESC Working Group on Cellular Biology of the Heart (2016) and on Cardiovascular Regenerative and Reparative Medicine (2017). In 2019, she became a member of the ESC Scientists of Tomorrow Nucleus. 